\title{
Variabilidad climática en las ciudades urbanas de Tegucigalpa y Comayagüela, Francisco Morazán, Honduras en el periodo comprendido entre los años $1975-2011$
}

Antonio Carías

\section{Resumen}

En este trabajo se estudia la variabilidad climática urbana del Distrito Central en Honduras lo cual comprende a Tegucigalpa la capital y a Comayagüela como ciudad vecina, el espacio temporal del análisis abarca las fechas de 1975, 1987, 2000 y 2011. En la primera etapa se estudia el patrón de crecimiento urbano lo que nos muestra que han ocurrido varios cambios en los primeros años de estudio, esta etapa del análisis se elaboró mediante imágenes satelitales del sensor LandSat, a estas imágenes se les calculo el índice de vegetación (NDVI) el cual separa las zonas vegetales de las desprovistas de vegetación lo cual nos hace un extracto de las zonas urbanas y así extraerlas con mayor facilidad, luego se evaluó los resultados de los análisis de la banda 6 del satélite LandSat que corresponde a la banda térmica con la cual podemos sacar temperatura del suelo en el momento en que fue tomada la imagen, es importante mencionar que las imágenes corresponden al primer trimestre del año y en este análisis no contamos con el año 1975 ya que el sensor no tenía instalada esa capacidad en esos momentos; sumado a estos procesos se analizó ciertas estaciones de monitoreo atmosférico y se procesaron mediante geoestadística lo cual arrojo un dato no tan local ya que las estaciones estaban muy separadas para arrojar un análisis un poco más particular. La suma de todas estas variables es el principal aporte a este estudio para guiar de alguna manera estudios de la temática de variabilidad climática y crecimiento urbano.

Palabras clave: Clima, Geo-estadística, Sensores Remotos, Temperatura, Urbano. 


\section{Abstract}

In this paper we study the climatic variability in Honduras Central District which includes the capital Tegucigalpa and Comayagüela as neighboring city, temporary space analysis covers the dates 1975, 1987, 2000 and 2011. In the first stage examines urban growth pattern which shows that there have been several changes in the first years of study, this stage of analysis if produced by sensor Landsat satellite images, these images will be calculating the vegetation index (NDVI) which separates the plant areas devoid of vegetation which makes us an excerpt from urban and thus remove them more easily, then evaluated the results of the analysis of Landsat satellite band 6 which corresponds to the thermal band with which we can draw soil temperature at the time the image was taken, it is important to mention that the images correspond to the first quarter of the year and in this analysis do not have the year 1975 because the sensor was not installed that capacity at the time; joined these analyzes was analyzed certain atmospheric monitoring stations and worked through geostatistics which throw a figure not so local as the stations were too far apart to shed a little more specific analysis. The sum of all these variables is the main contribution of this study to guide studies somehow the topic of climatic variability and urban growth.

Key words: Climate, Geo-statistics, Remote Sensing, Temperature, Uraban.

Antonio Carias, Departamento de Ciencia y Tecnologías de la Información Geográfica, Facultad de Ciencias Espaciales, Universidad Nacional Autónoma de Honduras 


\section{Introducción}

La rápida urbanización de las comunidades en estos últimos 50 años ha generado un aumento a la exposición a desastres naturales. El crecimiento urbano avanza hacia la frontera con los recursos naturales, lo que implica la extracción de recursos, consumo de energía y aumento de la polución ambiental. Esta degradación ambiental que ocurre en estas áreas urbanas incrementa los contaminantes atmosféricos lo cual aumenta los niveles de calor lo cual incrementa el consumo de energía en la utilización de sistemas de enfriamiento, estos impactos sumados a las demás ciudades se reflejan a escalas globales alterando los patrones de lluvias, la presencia de gases de invernadero, alteración de la humedad y de la disponibilidad de agua potable.

Es por ello que es necesario contar con una cuantificación de la expansión urbana y así mismo hacer una relación entre expansión y su comportamiento con el clima, para promover medidas que nos lleven a mitigar estos impactos ambientales que deterioran la salud de toda la biología circundante.

\section{Materiales y métodos.}

En los últimos años el estudio de asentamientos humanos y su respectivo crecimiento ha generado un notable interés, es por ello que se han generado diversos modelos espaciales utilizados por los urbanistas y ambientalistas para buscar el desarrollo de las regiones. (Herold, 2005)

Esta necesidad de establecer o descubrir estas tendencias, se hacen más fácil mediante los usos y procesamiento computacional con sistemas de información geográfica, esta es una herramienta vital para estos análisis ya que ella arroja resultados para las futuras tomas de decisiones. Para las interpretaciones territoriales es necesario contar con imágenes satelitales de la zona de estudio, como parte del modelo a trabajar en la planificación. Una caracterización territorial mediante sensores remotos es una técnica que acelera el dinamismo de la investigación espacial del crecimiento urbano esta da una diferente visión del territorio desde las regiones del espectro electromagnético y procesarlas de una fácil manera mediante medios digitales. (Lu, 2004)

La Municipalidad del Distrito Central comprende las ciudades de Tegucigalpa y Comayagüela, que hasta 1938 tenían organización administrativa independiente como municipios. En el Decreto 2, promulgado por el Honorable Congreso Nacional el 9 de diciembre de 1937, se ratifica el Decreto 53 del 30 de enero de 
1937, el cual declara en el Artículo 1. Reformar el Artículo 179 de la Constitución Política de 1936, en el que se dice que los actuales Municipios de Tegucigalpa y de Comayagüela, formarán un Distrito central, cuya creación, organización y funcionamiento será objeto de una ley especial.

En la actualidad, el Distrito Central ocupa un área de $1396.5 \mathrm{~km} 2$, divididos en un sector urbano (127.35 km2) y el resto en un sector rural (44 aldeas y 294 caseríos). La denominación municipal no corresponde a lo urbano, por el contrario, los municipios incluyen mayoritariamente áreas rurales y un número de poblados que se clasifican en escala desde aldeas y caseríos hasta áreas metropolitanas.

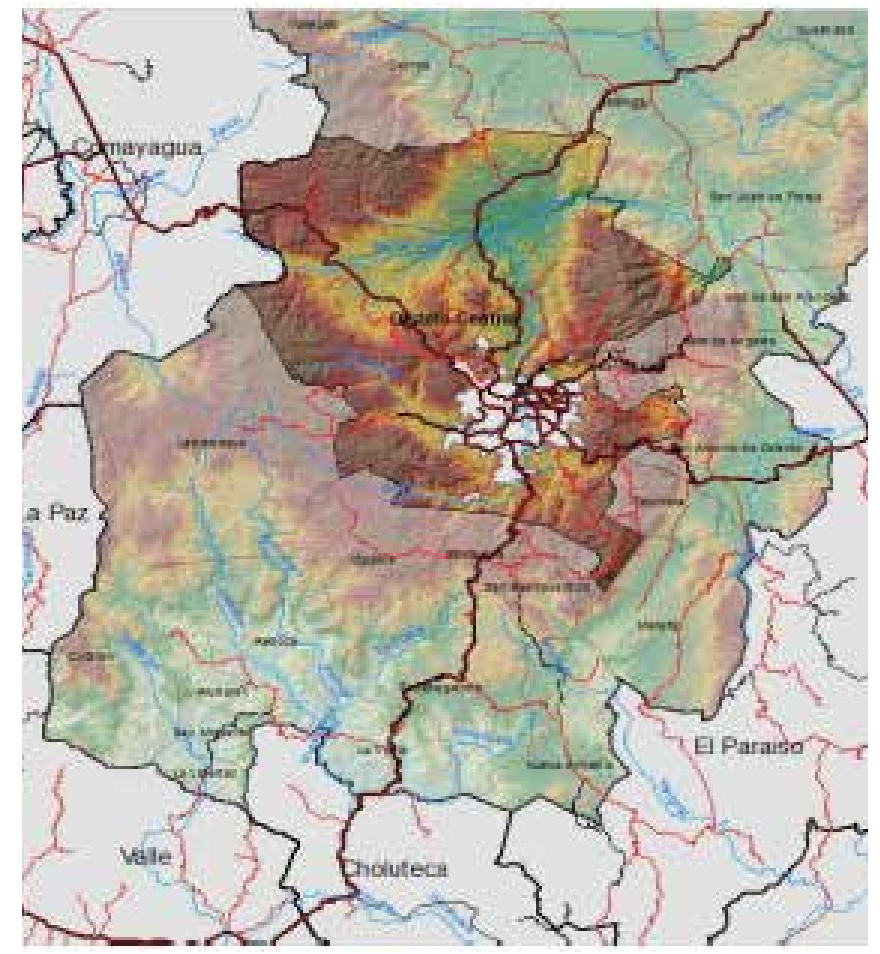

Figura 1 Delimitación del área de estudio

La ciudad de Tegucigalpa y sus municipios vecinos se encuentra ubicada en la Cuenca Alta del Río Choluteca, que vierte hacia el océano pacifico y que en su totalidad abarca unos $6510 \mathrm{~km} 2$. En la actualidad esta cuenca como sus principales subcuencas (Guacerique, Rio Grande, Sabacuante, Tatumbla y Río del Hombre) se encuentran en un fuerte proceso de degradación. 
La topografía del territorio tiene un rango de pendiente entre el 16 y el $30 \%$, con características geomorfológicas de bajo a alto riesgo, suelos poco profundos, que sumados a las fallas geológicas existentes da lugar a terrenos inestables.

Tiene un clima tropical lluvioso en las partes bajas, y templado húmedo en las partes altas. Por ubicarse en un altiplano situado a una altura entre los $900 \mathrm{y}$ 1050 metros sobre el nivel del mar (msnm), en el arranque de una cadena montañosa de la que forman parte los cerros El Berrinche, El Picacho y el Pedregal, presenta un clima templado con temperaturas promedio mensuales que varían desde los 19.4 grados centígrados en diciembre hasta los 23.5 grados en Mayo.

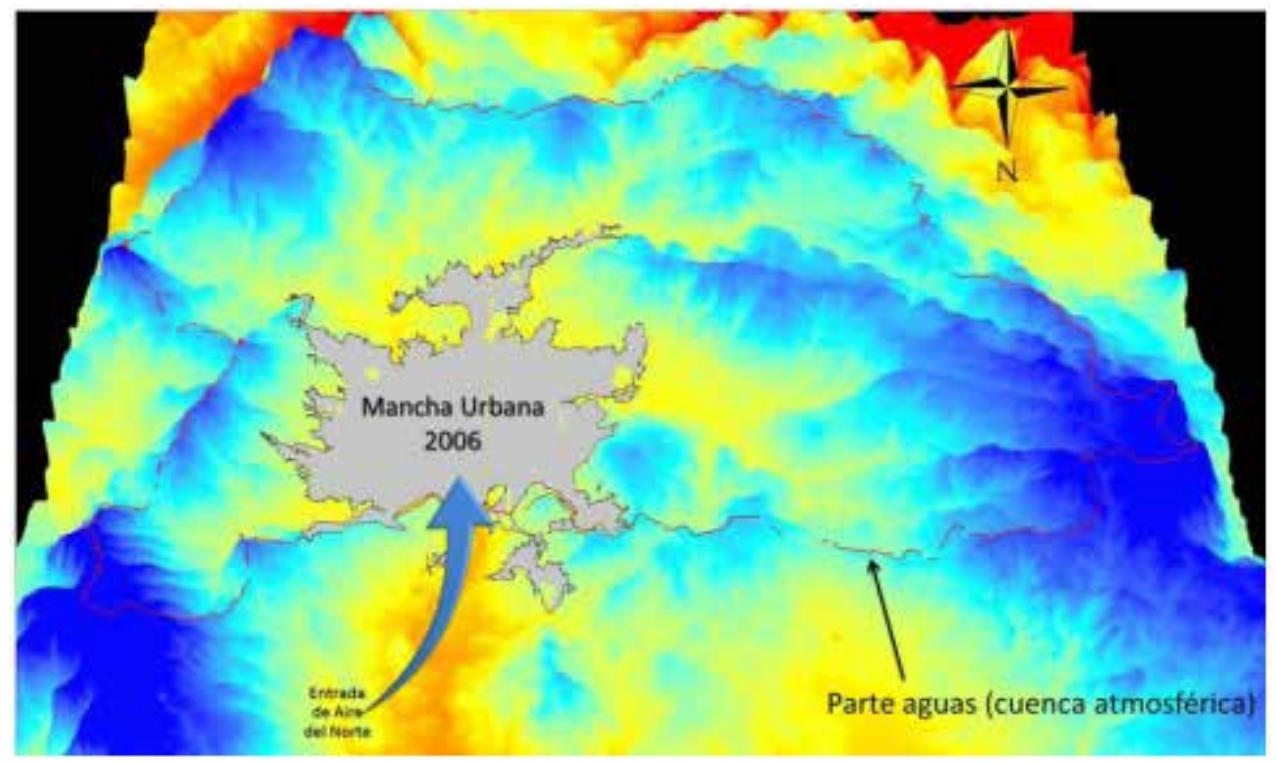

Figura 2 Cadena montañosa formando la cuenca atmosférica

En los últimos 25 años, el área urbana de Tegucigalpa se ha triplicado en tamaño. La mayor parte de esta expansión se produjo entre mediados de 1970 y finales de 1980. Impulsado por el rápido crecimiento de la población, el área urbana de la ciudad creció de aproximadamente 2.360 hectáreas en 1975 a 6.020 hectáreas en 1987. Durante este período, la ciudad experimentó la disminución de la densidad de población, ya que el por ciento de crecimiento en la expansión urbana (155\%) superó el por ciento de crecimiento de la población (83\%).

En 1987 el consumo per cápita de la tierra había crecido a más de 0,01 ha / persona de aproximadamente 0.007 ha / persona en 1975. Tegucigalpa continuó 
creciendo entre 1987 y 2000, la expansión de aproximadamente 2.340 hectáreas. Sin embargo a diferencia del período anterior, el porcentaje de crecimiento de la expansión urbana $(39 \%)$ fue sólo ligeramente inferior al porcentaje de crecimiento de la población (42\%). El aumento de la densificación en este período se hizo evidente en la caída de $2 \%$ en el consumo de suelo per cápita, pasando de 0,0103 ha I persona en 1987 hasta 0,0101 ha / persona en 2000 (Princeton University, 2004).
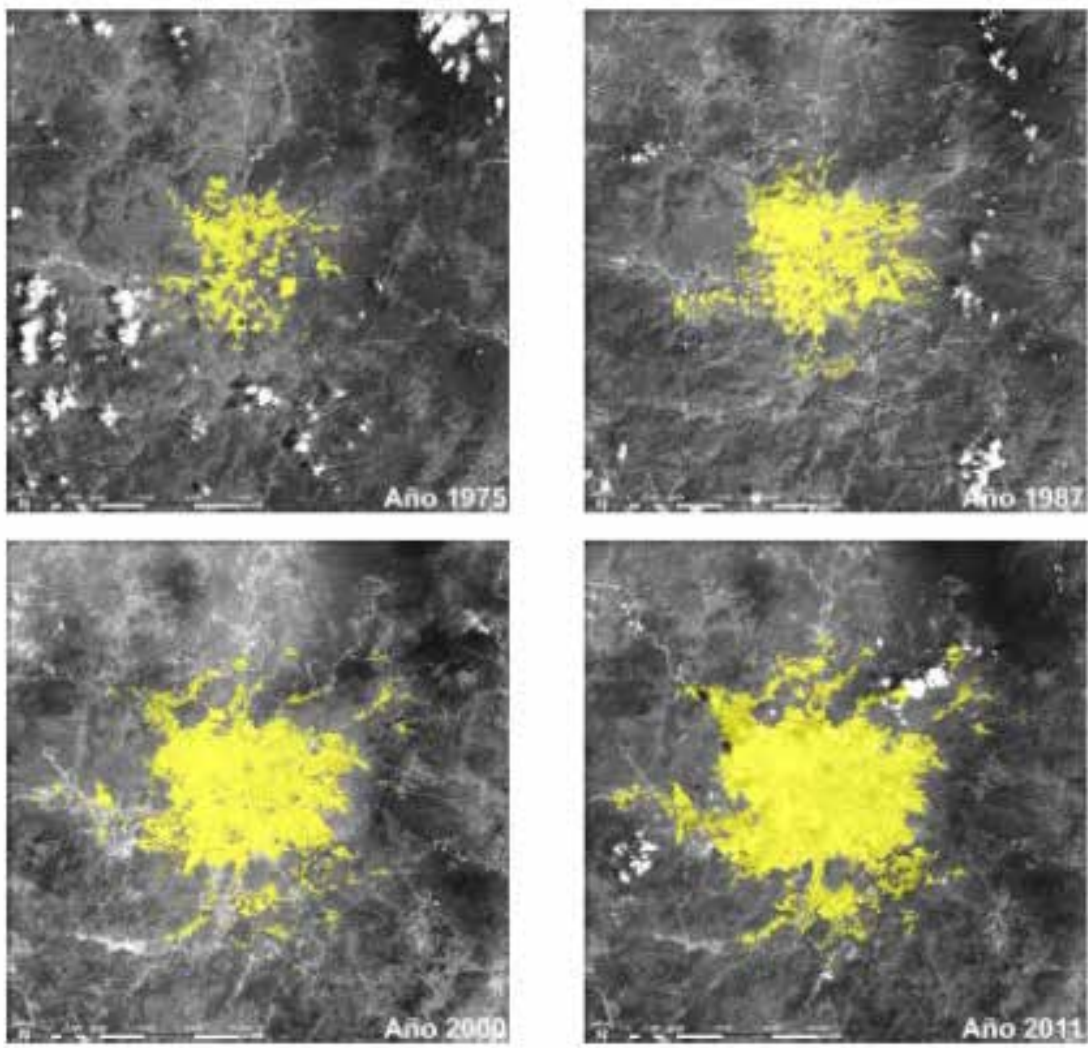

Figura N

\section{Marco Metodológico}

Las imágenes que se trabajaron fueron unas LandSat TM adquiridas para los años de 1975, 1987, 2000, 2011 todas ellas pertenecientes al primer trimestre de cada año, tiene una resolución espacial de 30 metros y la termal de 120 metros. 
Primero hay que obtener las reflectancias de la superficie de estudio luego de pre procesar y calibrar dichas radiaciones atmosféricas y correcciones geométricas, se obtiene la imagen mediante un corte procesado de radiometría.

\section{Obteniendo el NDVI}

Teniendo la imagen principal con las correcciones correspondientes se procede a extraer el NDVI (Índice de vegetación de diferencia normalizada) el cual su valor mínimo es el valor del suelo puro o desprovisto de vegetación. La emisión de la superficie es calculada mediante la siguiente formula:

$$
\varepsilon=0.98-0.042 \rho
$$

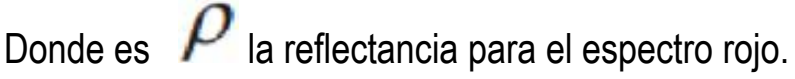

Y cuando el valor del NDVI es máximo significa que el pixel demuestra pura vegetación y la emisión de la superficie es igual a 0.986.

Cuando el valor del NDVI es menor o igual al NDVI máximo y mayor o igual al NDVI mínimo el valor de pixel es una mezcla y esta se deriva de la siguiente formula:

$$
\varepsilon=\varepsilon_{v} P_{v}+\varepsilon_{s}\left(1-P_{v}\right)+d \varepsilon
$$
y suelo.

Los parámetros $\varepsilon_{v \mathrm{v}} \mathcal{E}_{s}$ representan las reflectancias de la vegetación

Expresa la diferencia de reflectancia causada por la distribución geométrica del efecto superficial y está en muchos casos el valor el cero.

Refiere a la composición de vegetación expresada por la fórmula:

$$
P_{v}=\left[\frac{N D V I-N D V I_{\min }}{N D V I_{\max }-N D V I_{\min }}\right]^{2}
$$




\section{Obteniendo la Temperatura de la Superficie del Terreno (LST)}

Para calcular la temperatura de brillo de la radiación (_ ) que está compuesta por la emisión de calor del objeto y energía de la radiación del ambiente expresada como la diferencia de la temperatura del suelo se calcula con la siguiente formula:

$$
L_{\lambda}=(\text { Gain } * D N)+\text { Bias }
$$

En esta fórmula los datos de Gain y Bias son adoptados del archivo cabecera de la imagen satelital, él es el numero digital que representa el valor numérico de cada pixel en la imagen.

Para calcular la temperatura $T_{d}$ del suelo se utiliza la siguiente ecuación (Wukelic, 1989):

$$
T_{d}=K_{2} / \ln \left(1+k_{1} / L_{\lambda}\right)
$$

Los parámetros $\mathrm{K} 1$ y K2 de la ecuación son valores constantes en grados Kelvin para cada uno respectivamente $60.776 \mathrm{~K}$ y $1260.56 \mathrm{~K}$.

Calculando la temperatura de la superficie del terreno (David AArtis, 1982):

$$
T_{s}=T_{d} / 1+(\lambda / \rho) \ln \varepsilon
$$

Importante recalcar que el resultado que obtenemos en $P_{v}$ la es en grados Kelvin por lo cual debemos convertirlo a grados Centígrados usando esta fórmula:

$$
T=T s-273
$$




\section{Obteniendo mapa geo estadístico mediante información ambiental}

Para generar dichos mapas se partió de información de estaciones meteorológicas las cuales fueron procesadas para generar el Índice de Severidad de Sequia (ISS) los cuales fueron interpolados mediante La media ponderada por el inverso de la distancia, denominada en inglés inverse distance waited (IDW), asume que hay una relación entre distancias, los valores cercanos a un punto tienen más parecido que los que están más lejos. (JOHNSTON et al., 2001)

Para predecir un valor de un lugar no muestral, utilizara los valores de los lugares muestrales que haya alrededor del lugar que se va a predecir.

Los valores de los lugares más próximos al que se va a predecir tendrán más influencia y por lo tanto más peso que los que están más lejos. Este peso disminuye con la distancia. La fórmula general es:

$$
Z\left(S_{0}\right)=\sum_{i=1}^{N} \lambda * Z\left(S_{i}\right)
$$

Donde $Z\left(S_{0}\right)$ es el valor que intentamos predecir para el lugar $S_{0} . \mathrm{N}$ es el número de puntos muestrales alrededor del lugar que se va a predecir y que serán tenidos en cuenta en la predicción. $\lambda_{i}$ es el peso asignado a cada punto muestral que vamos a usar. Estos pesos decrecen con la distancia. $Z$ ( $\left.\mathrm{S}_{\mathrm{i}}\right)$ es el valor observado del lugar $\mathrm{S}_{\mathbf{j}}$.

La fórmula para determinar los pesos es:

$$
\lambda_{i}=d_{i 0}^{-p} / \sum_{i=1}^{n} d_{i 0}^{-p}
$$

A medida que la distancia se hace más grande, el peso es reducido por un factor $p$. Es decir, que a medida que se incrementa la distancia entre los puntos observados y el punto calculado, el peso que tendrá un punto muestral sobre el predicho decrecerá exponencialmente. dio es la distancia entre el lugar de predicción SO y cada lugar muestral, Si. Los pesos de los lugares utilizados para la predicción serán escalados de tal forma que su suma sea 1, es decir,

$$
\sum_{i=1}^{N} \lambda_{i}=1
$$


El óptimo valor de $p$ se determina haciendo mínimo el error de predicción 0 error cuadrático medio, que tiene la siguiente fórmula:

$$
E M C=\sqrt{\sum_{i=0}^{n} \frac{\left(\mathcal{Z}\left(s_{i}\right)-z\left(s_{i}\right)\right)^{2}}{n}}
$$

El analista geoestadístico utiliza potencias más grandes que 1. Por defecto usa $\quad p=2$ que se conoce como distancia inversa al cuadrado.

Otra cuestión importante es determinar el número de vecinos a tener en cuenta para calcular el valor predicho. Esto dependerá del tipo de datos y de la superficie que intentemos crear.

La superficie calculada usando la media ponderada por el inverso de la distancia (IDW) dependerá de la potencia del parámetro $\mathrm{p}$ y de la estrategia de búsqueda de vecindad. El IDW es un interpolador exacto, donde los valores máximos y mínimos en la superficie interpolada pueden solamente ocurrir en los puntos muéstrales.

\section{Resultados}

Los resultados obtenidos del procedimiento LST corresponden a las fechas de los años 1987, 2000 y 2011 todos pertenecientes al primer trimestre del año respectivo los cuales se muestran a continuación: 

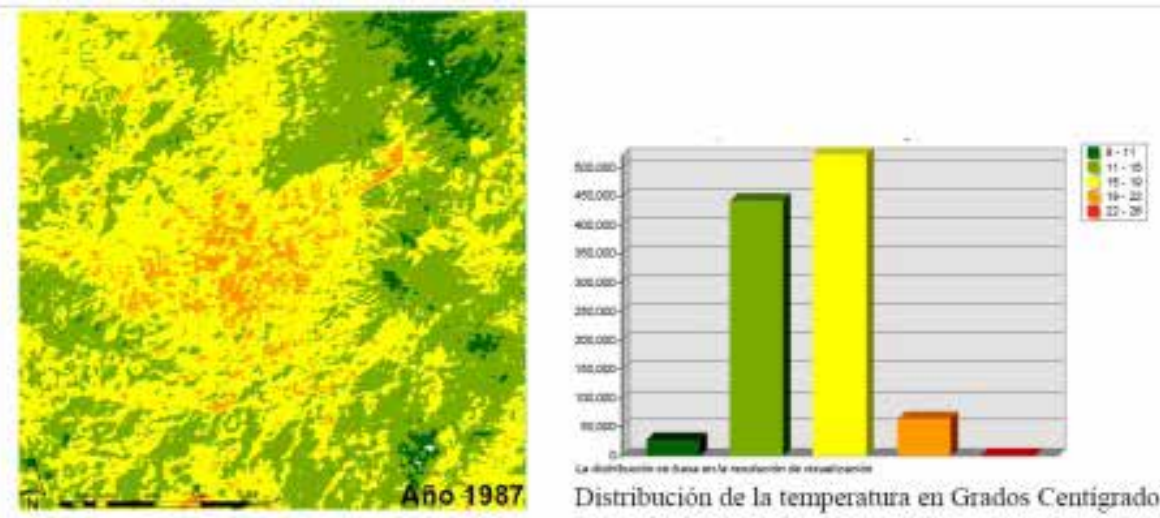

Distribución de la temperahura en Grados Centigrados
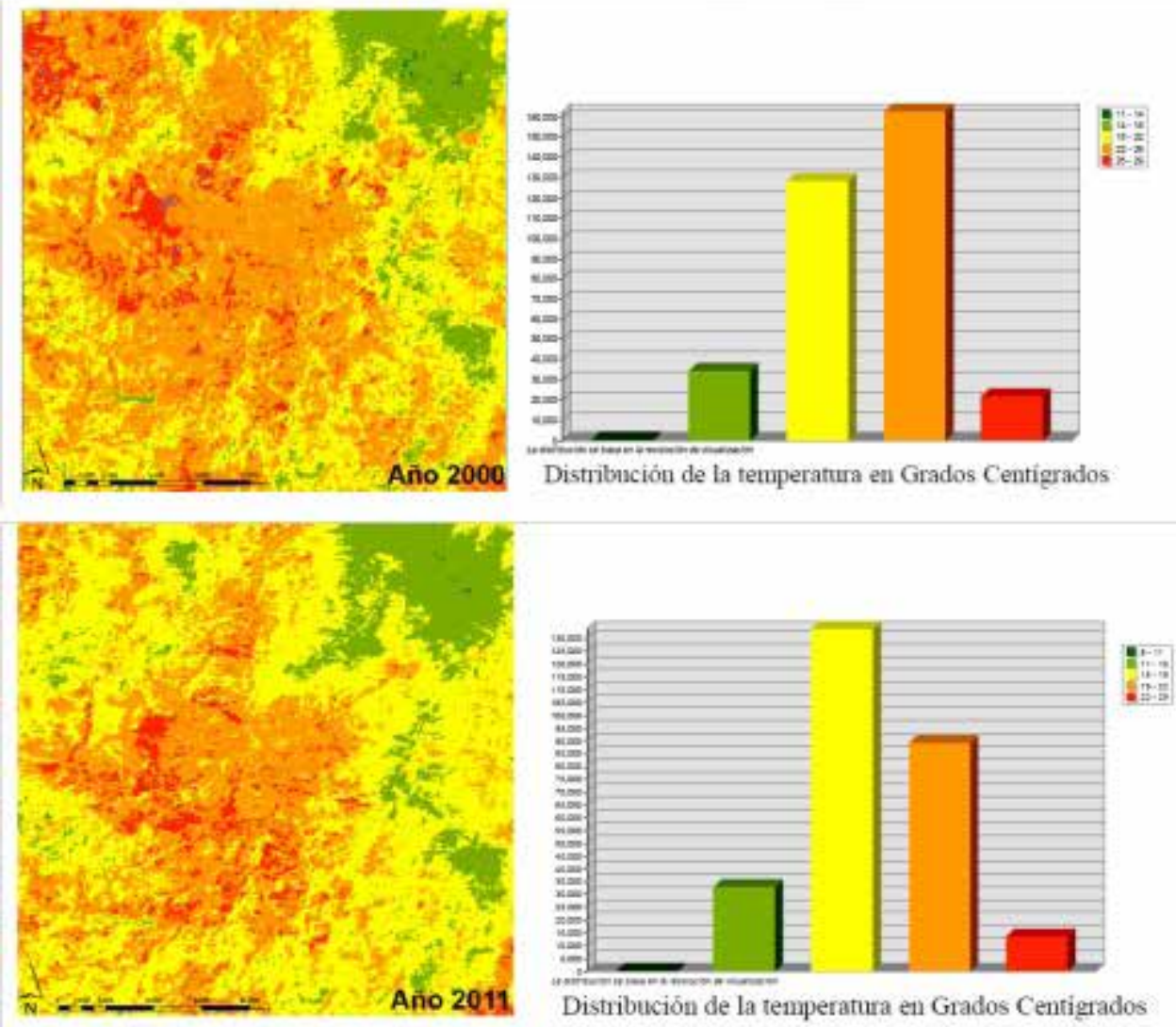

Como se muestra en las gráficas junto al mapa vemos para el año 1987 que el comportamiento de la temperatura está dentro de los rangos de $11^{\circ} \mathrm{C}-19^{\circ} \mathrm{C}$ par el año 2000 están entre $18^{\circ} \mathrm{C}-26^{\circ} \mathrm{C}$ y para el año 2011 entre $15^{\circ} \mathrm{C}-22^{\circ} \mathrm{C}$ 
Utilizando los métodos estadísticos de interpolación IDW tenemos los siguientes mapas mostrando las zonas con un índice de severidad de sequía el cual fue desarrollado para medir la intensidad, duración y extensión espacial de la sequía, estos valores derivan de las medidas de precipitación, temperatura del aire y humedad del suelo local.
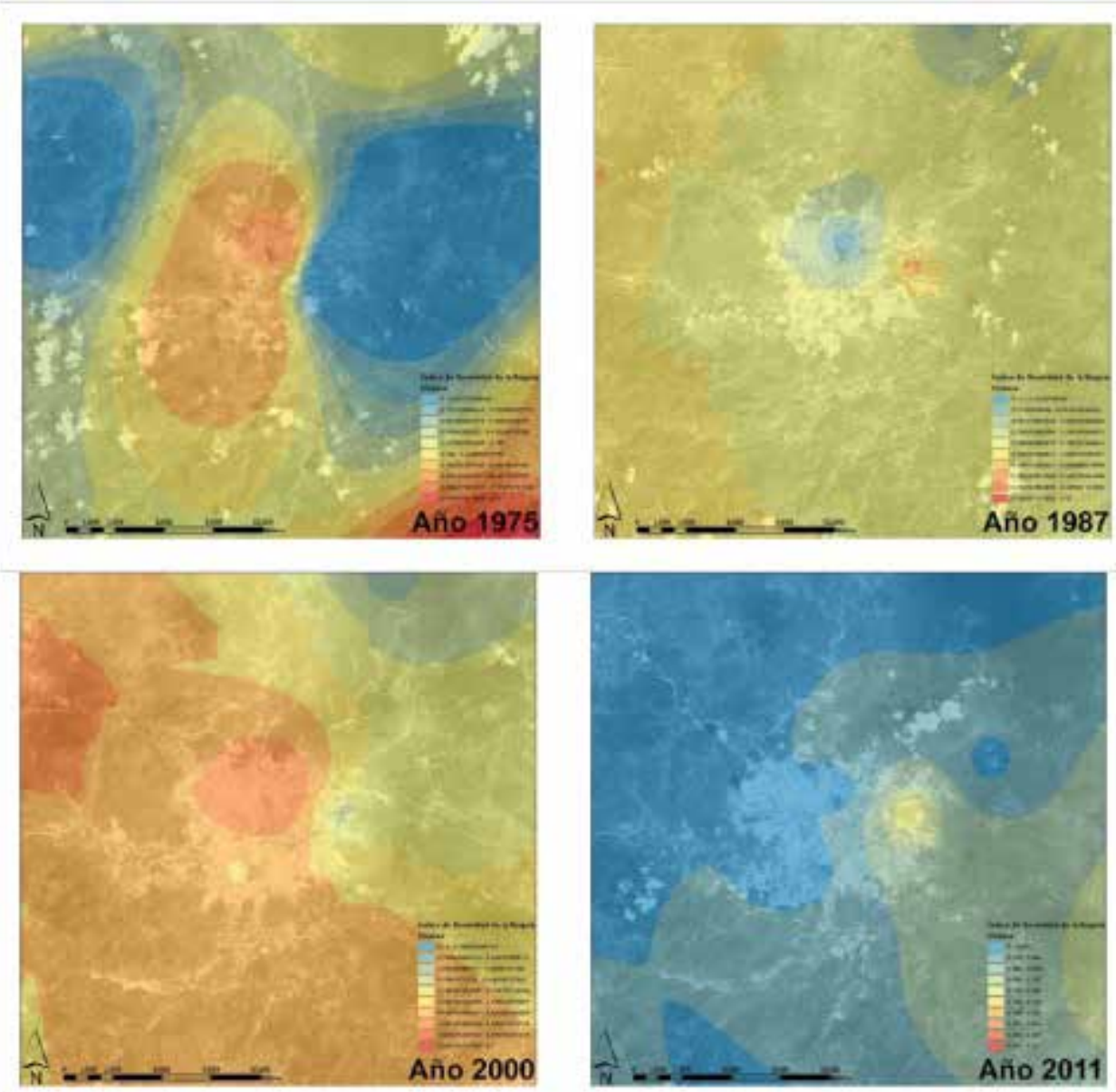

\section{Conclusiones}

Con los estudios obtenidos con las diferentes modelos (NDVI, LST e IDW), sus respectivas comparaciones, y a pesar de que los espacios temporales son considerables se deduce que el crecimiento del Distrito Central ha afectado en medida y se estima de que hay una tendencia aparente de un aumento de temperatura en 
los pasados 35 años y las correlaciones con los diferentes modelos estadísticos corresponden en todos los años.

Cabe destacar que en los últimos años se muestran un descenso de la temperatura ya sea en el análisis estadístico como en el cálculo de temperatura de superficie, lo cual de alguna manera satisface el estudio ya que vemos una correlación positiva en ellos.

Es importante contar con programas de revegetación urbana, programas educativos de la gestión del riesgo, fortalecer los planes de ordenamiento territorial correspondientes a planeamiento urbano ecológico, tratar de reducir al máximo los efectos negativos que incurre en un avance urbanístico sin planificación ecológica para guardar la salud de los ciudadanos y los peligros naturales ya sean epidémicos o de polución.

\section{Bibliografía.}

- Barredo, J. I. (2003). Modeling dynamic spatial processes: simulation of urban future scenarios trough cellular automata. En Landscape and Urban Planning (págs. 145-160).

- David A Artis, W. H. (September de 1982). Survey of emissivity variability in thermography of urban areas. Terre Haute, Indiana, U.S.A.

- Herold, M. H. (2005). The role of spatial metrics in the analysis and modeling of land use change. Environments and Urban Systems, 369-399.

- Jantz, C. A. (2003). Using the SLEUTH urban growth model to simulate the impacts of future policy scenarios on urban Land use in the Baltimore metropolitan area. Washington.

- JOHNSTON et al. (2001). Using ArcGIS geostatical analyst. New York: ESRI.

- Lu, D. P. (2004). Change detection techniques. International Journal of Remote Sensing, 2365-2407.

- Princeton University. (February de 2004). Rapid Urbanization in Tegucigalpa, Honduras. Princeton, NJ. 
- White, Engelen R., y G. Uljee. (1997). The use of constrained cellular automata for high resolution modeling of urban land dynamics. En Environmental Plannig (págs. 323-343).

- Wukelic, G. E. (1 de Jun de 1989). Radiometric calibration of Landsat Thematic Mapper Thermal Band. Richland, United States. 